\title{
CONVERGENCE OF THE SQUARE ROOT ENSEMBLE KALMAN FILTER IN THE LARGE ENSEMBLE LIMIT
}

\author{
EVAN KWIATKOWSKI* AND JAN MANDEL*†
}

\begin{abstract}
Ensemble filters implement sequential Bayesian estimation by representing the probability distribution by an ensemble mean and covariance. Unbiased square root ensemble filters use deterministic algorithms to produce an analysis (posterior) ensemble with a prescribed mean and covariance, consistent with the Kalman update. This includes several filters used in practice, such as the Ensemble Transform Kalman Filter (ETKF), the Ensemble Adjustment Kalman Filter (EAKF), and a filter by Whitaker and Hamill. We show that at every time index, as the number of ensemble members increases to infinity, the mean and covariance of an unbiased ensemble square root filter converge to those of the Kalman filter, in the case of a linear model and an initial distribution of which all moments exist. The convergence is in all $L^{p}, 1 \leq p<\infty$, and the convergence rate does not depend on the model or data dimensions. The result holds in infinitely dimensional Hilbert spaces as well.
\end{abstract}

Key words. Data assimilation, $L^{p}$ laws of large numbers, Hilbert space, ensemble Kalman filter

AMS subject classifications. 60F $25,65 \mathrm{C} 05,65 \mathrm{C} 35$

1. Introduction. Data assimilation uses Bayesian estimation to incorporate observations into the model of a physical system. The model produces the forecast estimate, and the incorporation of the observation data produces the analysis estimate. The resulting analysis is used to initialize the next run of the model, producing the next forecast, which subsequently is used in the next analysis, and the process thus continues. Data assimilation is widely used, e.g., in geosciences 17.

The Kalman filter represents probability distributions by the mean and covariance. It is an efficient method when the probability distributions are close to Gaussian. However, in applications, the dimension of the state is large, and it is not feasible to even store the covariance of the system state exactly. Ensemble Kalman filters are variants of Kalman filters in which the state probability distribution is represented by an ensemble of states, and the state mean and covariance are estimated from the ensemble [12. The dynamics of the model, which could be nonlinear in this case, are applied to each ensemble member to produce the forecast estimate. The simplest estimate of covariance from the ensemble is the sample covariance, which, however, suffers from sampling errors for small ensembles. For this reason, localization techniques, such as tapering [14] and covariance inflation [3, 4, need to be used for small ensembles [12, Ch. 15]. Simulation studies have shown that the ensemble filters with relatively small ensembles and with localization and covariance inflation are able to efficiently handle nonlinear dynamics and high dimension.

The major differences between ensemble Kalman filters are in the way the analysis ensemble is produced from the forecast and the data. The analysis ensemble can be formed in a stochastic or deterministic manner. The purpose of this paper is to examine ensemble Kalman filters that use a deterministic method to produce an analysis ensemble with exactly the desired statistics. Such filters are called unbiased square root filters, because the ensemble mean equals the prescribed mean, and construction of the analysis ensemble to match the prescribed ensemble covariance leads to taking the square root of a matrix. This includes several filters used in practice, such as the Ensemble Transform Kalman Filter (ETKF) 66, 15, 33, the Ensemble Adjustment Kalman Filter (EAKF) 2, and a filter by Whitaker and Hamill 34. Criteria necessary for an ensemble square root filter to be unbiased are discussed in 23, 30, 32. The base square root ensemble Kalman filter (cf., Algorithm 3.2 below) is often modified to support localization and covariance inflation for small ensembles and nonlinear problems. Since we are interested in large ensemble asymptotics and the linear case, localization and covariance inflation are not studied in this paper.

An important question for understanding ensemble filters is a law of large numbers as the size of the ensemble grows to infinity, even if practical implementations are necessarily limited to small ensembles. In 22, 24, it was proved independently for the version of the ensemble Kalman filter with randomized data from [8, that the ensemble mean and the covariance matrix converge to those of the Kalman filter, as the

\footnotetext{
${ }^{*}$ Department of Mathematical and Statistical Sciences, University of Colorado Denver, Denver, CO, USA. Partially supported by the U.S. National Science Foundation under the grant DMS-1216481.

${ }^{\dagger}$ Institute of Computer Science, Academy of Sciences of the Czech Republic, Prague, Czech Republic. Partially supported by the Czech Science Foundation under the grant 13-34856S.
} 
number of ensemble members grows to infinity. Both analyses obtain convergence in all $L^{p}, 1 \leq p<\infty$, but the convergence results are not independent of the space dimensions. The proof in [24] relies on the fact that ensemble members are exchangeable, uses uniform integrability, and does not provide any convergence rates, while 22 uses stochastic inequalities for the random matrices and vectors to obtain convergence with the usual Monte Carlo rate $1 / \sqrt{N}$, but relies on entry-by-entry arguments.

Here, we show that at every time index, as the number of ensemble members increases to infinity, the mean and covariance of an unbiased ensemble square root filter converge to those of the Kalman filter, in the case of a linear model and an initial distribution of which all moments exist. The convergence is in all $L^{p}$, with the usual rate $1 / \sqrt{N}$, the constant does not depend on the dimension, and the result holds in the infinitely dimensional case as well. The constants in the estimate are constructive and depend only on the model and the data, namely the norms of the model operators and of the inverse of the data covariance, and of the vectors given. The analysis builds on some of the tools developed in [22, and extends them to obtain bounds on the operators involved in the formulation of square root ensemble filters, independent of the state space and data space dimensions, including the infinitely dimensional case. The square root ensemble filters are deterministic, which avoids technical complications associated with data perturbation in infinite dimension. Convergence of ensemble filters with data perturbation, independent of the dimension, will be studied elsewhere.

The main idea of the analysis is simple: by the law of large numbers, the ensemble mean and covariance of the initial ensemble converge to those of the background distribution. Every analysis step is a continuous mapping of the mean and the covariance, and the convergence in the large ensemble limit follows. The analysis quantifies this argument.

Since the principal observation is that the mean and the covariance are transformed in exactly the same way as in the Kalman filter, the continuity estimates in this paper can also be interpreted as a type of stability of the Kalman filter for arbitrary but fixed time with respect to perturbations of the initial distribution. The continuity of the Kalman filter formulas is foundational, and, as such, has not received much attention. The pointwise estimates in the present paper are somewhat stronger, and they imply local Lipschitz continuity with polynomial growth of the constant. The $L^{p}$ estimates can be interpreted as the stability of each Kalman filter time step separately with respect to random perturbations of the initial mean vector and covariance operator. This type of stability of the Kalman filter seems not to have been studied before. However, there has been a keen interest in long-time stability, particularly the effect of a finite error in the initial distribution diminishing over time, both theoretically for the Kalman filter (e.g., [21, 28, 37]) and empirically for the ensemble Kalman filter in meteorology (e.g., 36]). For a class of abstract dynamical systems with the whole state observed, the ensemble Kalman filter with a fixed ensemble size was proved to be well-posed and not blowing up faster than exponentially, and to stay within a bounded distance from the truth if sufficiently large covariance inflation is used [18].

The paper is organized as follows: in Section 2 we introduce notation and review select background concepts. Section 3 contains statements of the Kalman filter and the unbiased square root filter, and shows some basic properties, which are needed later. In Section 4, we show the continuity properties of the transformation of the statistics from one time step to the next. Section 5 contains a derivation of the $L^{p}$ laws of large numbers, needed for the convergence of the statistics of the initial ensemble. Section 6 presents the main result.

2. Notation and preliminaries. We will work with random elements with values in a Hilbert space $V$. Readers interested in finite dimension only can think of random vectors in $V=\mathbb{R}^{n}$. All notations and proofs are presented in a way that applies in $\mathbb{R}^{n}$, as well as in a more general Hilbert space.

2.1. Finite dimensional case. Vectors $u \in \mathbb{R}^{n}$ are columns, and the inner product is $\langle u, v\rangle=u^{*} v$ where $u^{*}$ denotes transpose, and $|u|=\langle u, u\rangle^{1 / 2}$ is the vector norm of $u$. Throughout this paper, we will use single bars $|\cdot|$ for deterministic norms and double bars $\|\cdot\|$ for stochastic norms. We will use the notation $[V]$ for the space of all $n \times n$ matrices. For a matrix $M, M^{*}$ denotes transpose, and $|M|$ stands for the spectral norm. We will also need the Hilbert-Schmidt norm (more commonly called Frobenius norm) of matrices, 
induced by the corresponding inner product of two $n \times n$ matrices,

$$
|A|_{\mathrm{HS}}=\left(\sum_{i, j=1}^{n} a_{i j}^{2}\right)^{1 / 2}=\langle A, A\rangle_{\mathrm{HS}}^{1 / 2}, \quad\langle A, B\rangle_{\mathrm{HS}}=\sum_{i, j=1}^{n} a_{i j} b_{i j} .
$$

The Hilbert-Schmidt norm dominates the spectral norm,

$$
|A| \leq|A|_{\mathrm{HS}}
$$

for any matrix $A$. The notation $A \geq 0$ means that $A$ is symmetric and positive semidefinite, $A>0$ means symmetric positive definite. For $Q \geq 0, X \sim N(u, Q)$ means that random vector $X$ has the normal distribution on $\mathbb{R}^{n}$, with mean $u$ and covariance matrix $Q$. For vectors $u, v \in \mathbb{R}^{n}$, their tensor product is the $n \times n$ matrix,

$$
u \otimes v=u v^{*} \in[V] .
$$

It is evident that

$$
|u \otimes v|_{\mathrm{HS}}=|u||v|
$$

because

$$
|u \otimes v|_{H S}^{2}=\sum_{i=1}^{n} \sum_{j=1}^{n}\left(u_{i} v_{j}\right)^{2}=\sum_{i=1}^{n} u_{i}^{2} \sum_{j=1}^{n} v_{j}^{2}=|u|^{2}|v|^{2} .
$$

2.2. Hilbert space case. Readers interested in finite dimension only should skip this section. In the general case, $V$ is a separable Hilbert space equipped with inner product $\langle u, v\rangle$ and the norm $|u|=\langle u, u\rangle^{1 / 2}$. The space of all bounded operators from Hilbert space $U$ to Hilbert space $V$ is $[U, V]$, and $[V]=[V, V]$. For a bounded linear operator $A \in[U, V], A^{*} \in[V, U]$ denotes the adjoint operator, and $|A|$ is the operator norm. The Hilbert-Schmidt norm of a linear operator on $V$ is defined by

$$
|A|_{\mathrm{HS}}=\langle A, A\rangle_{\mathrm{HS}}^{1 / 2}, \quad\langle A, B\rangle_{\mathrm{HS}}=\sum_{i=1}^{\infty}\left\langle A e_{i}, B e_{i}\right\rangle
$$

where $\left\{e_{i}\right\}$ is any complete orthonormal sequence; the values do not depend on the choice of $\left\{e_{i}\right\}$. An operator on $V$ is called a Hilbert-Schmidt operator if $\langle A, B\rangle_{\mathrm{HS}}<\infty$, and $\mathrm{HS}(V)$ is the space of all HilbertSchmidt operators on $V$. The Hilbert-Schmidt norm again dominates the spectral norm, so (2.1) holds, and $\operatorname{HS}(V) \subset[V]$. The importance of $\operatorname{HS}(V)$ for us lies in the fact that $\operatorname{HS}(V)$ is a Hilbert space, while $[V]$ is not.

The notation $A \geq 0$ for operator $A \in[V]$ means that $A$ is symmetric, $A=A^{*}$, and positive semidefinite, $\langle A u, u\rangle \geq 0$ for all $u \in V$. The notation $A>0$ means here that $A$ is symmetric and bounded below, that is, $\langle A u, u\rangle \geq \alpha|u|^{2}$ for all $u \in V$ and some $\alpha>0$. In particular, if $A>0$, then $A^{-1} \in[V]$. For $A$, $B \in[V], A \leq B$ means that $A$ and $B$ are symmetric, and $B-A \geq 0$. It is well known from spectral theory that $0 \leq A \leq B$ implies $|A| \leq|B|$. Let $\rho(A)$ denote the spectral radius of $A$, and if $A$ is symmetric, then $\rho(A)=|A|$. For a symmetric operator $A \geq 0$, there exists a unique symmetric $A^{1 / 2} \geq 0$ such that $A^{1 / 2} A^{1 / 2}=A$, and if $A>0$, then also $A^{1 / 2}>0$ and $A^{-1 / 2}=\left(A^{1 / 2}\right)^{-1} \in[V]$.

An operator $A \geq 0$ is trace class if $\operatorname{Tr} A<\infty$, where $\operatorname{Tr} A$ is the trace of $A$, defined by

$$
\operatorname{Tr} A=\sum_{i=1}^{\infty}\left\langle A e_{i}, e_{i}\right\rangle
$$

If $A \geq 0$ is trace class, then $A$ is Hilbert-Schmidt, because $|A|_{\mathrm{HS}} \leq \operatorname{Tr} A$.

For vectors $u, v \in V$, their tensor product $u \otimes v \in[V]$ is now a mapping defined by

$$
u \otimes v: w \in V \mapsto u\langle v, w\rangle,
$$


and the proof of (2.2) becomes

$$
|x \otimes y|_{H S}^{2}=\sum_{i=1}^{\infty}\left|(x \otimes y) e_{i}\right|^{2}=\sum_{i=1}^{\infty}\left|x\left\langle y, e_{i}\right\rangle\right|^{2}=|x|^{2} \sum_{i=1}^{\infty}\left|\left\langle y, e_{i}\right\rangle\right|^{2}=|x|^{2}|y|^{2},
$$

from Bessel's equality.

The mean of a random element $E(X) \in V$ is defined by

$$
\langle E(X), y\rangle=E(\langle X, y\rangle) \quad \text { for all } y \in V .
$$

The mean of the tensor product of two random elements is defined by

$$
\langle E(X \otimes Y) w, y\rangle=E(\langle X, y\rangle\langle Y, w\rangle) \quad \text { for all } w, y \in V .
$$

The covariance of a random element $X$ (defined below in (2.3) $)$ exists when the second moment $E\left(|X|^{2}\right)<\infty$, and the proposed covariance is a trace class operator. On the other hand, if $Q \geq 0$ is trace class, the normal distribution $N(u, Q)$ can be defined as a probability measure on $V$, consistently with the finite dimensional case. Just as in the finite dimensional case, if $X \sim N(u, Q)$, then $X$ has all moments $E\left(|X|^{p}\right)<\infty$, $1 \leq p<\infty$. See [10, 19, 20] for further details.

2.3. Common definitions and properties. The rest of the background material is the same regardless if $V=\mathbb{R}^{n}$ or if $V$ is a general Hilbert space. To unify the nomenclature, matrices are identified with the corresponding operators of matrix-vector multiplication. Covariance of a random vector $X \in V$ is defined by

$$
\begin{aligned}
\operatorname{Cov}(X) & =E((X-E(X)) \otimes(Y-E(Y))) \\
& =E(X \otimes Y)-E(X) \otimes E(Y),
\end{aligned}
$$

if it exists. For $1 \leq p<\infty$, the space of all random elements $X \in V$ with finite moment $E\left(|X|^{p}\right)<\infty$ is denoted by $L^{p}(V)$, and it is equipped with the norm $\|X\|_{p}=\left(E\left(|X|^{p}\right)\right)^{1 / p}$. If $1 \leq p \leq q<\infty$ and $X \in L^{q}(V)$, then $X \in L^{p}(V)$ and $\|X\|_{p} \leq\|X\|_{q}$. If $X, Y \in L^{2}(V)$ are independent, then $\operatorname{Cov}(X, Y)=0$ and $E(\langle X, Y\rangle)=0$. The following lemma will be used repeatedly in obtaining $L^{p}$ estimates. It is characteristic of our approach to use higher order norms to bound lower order norms.

Lemma 2.1 ( $L^{p}$ Cauchy-Schwarz inequality). If $U, V \in L^{2 p}(V)$ and $1 \leq p<\infty$, then

$$
\||U||V|\|_{p} \leq\|U\|_{2 p}\|V\|_{2 p} .
$$

Proof. By the Cauchy-Schwartz inequality in $L^{2}(V)$, applied to the random variables $|U|^{p}$ and $|V|^{p}$, which are in $L^{2}(V)$,

$$
\begin{aligned}
\left\|\left|U\|V \mid\|_{p}^{p}\right.\right. & =E\left(|U|^{p}|V|^{p}\right) \leq\left(E|U|^{2 p}\right)^{1 / 2}\left(E|V|^{2 p}\right)^{1 / 2} \\
& =\left(\left(E|U|^{2 p}\right)^{1 / 2 p}\left(E|V|^{2 p}\right)^{1 / 2 p}\right)^{p}=\|X\|_{2 p}^{p}\|Y\|_{2 p}^{p} .
\end{aligned}
$$

Taking the $p$-th root of both sides yields the desired inequality. $\square$

An ensemble $\boldsymbol{X}_{N}$ consists of random elements $X_{i}, i=1, \ldots, N$. The ensemble mean is denoted by $\overline{\boldsymbol{X}}_{N}$ or $E_{N}\left(\boldsymbol{X}_{N}\right)$, and is defined by

$$
\overline{\boldsymbol{X}}_{N}=E_{N}\left(\boldsymbol{X}_{N}\right)=\frac{1}{N} \sum_{i=1}^{N} X_{i} .
$$

The ensemble covariance is denoted by $Q_{N}$ or $C_{N}\left(\boldsymbol{X}_{N}\right)$, and is defined by

$$
\begin{aligned}
Q_{N}=C_{N}\left(\boldsymbol{X}_{N}\right) & =\frac{1}{N} \sum_{i=1}^{N}\left(X_{i}-\overline{\boldsymbol{X}}_{N}\right) \otimes\left(X_{i}-\overline{\boldsymbol{X}}_{N}\right) \\
& =E_{N}\left(\boldsymbol{X}_{N} \otimes \boldsymbol{X}_{N}\right)-E_{N}\left(\boldsymbol{X}_{N}\right) \otimes E_{N}\left(\boldsymbol{X}_{N}\right),
\end{aligned}
$$


where

$$
\boldsymbol{X}_{N} \otimes \boldsymbol{X}_{N}=X_{1} \otimes X_{1}+\ldots+X_{N} \otimes X_{N}
$$

We use $N$ instead of the more common $N-1$, which would give an unbiased estimate, because it allows writing the sample covariance with the sample mean in (2.5) without the additional multiplicative factors $N /(N-1)$. Note that convergence as $N \rightarrow \infty$ is not affected.

3. Definitions and basic properties of the algorithms. In the Kalman filter, the probability distribution of the state of the system is described by its mean and covariance. We first consider the analysis step, which uses Bayes' theorem to incorporate an observation into the forecast state and covariance to produce the analysis state and covariance. The system state $X$ is an $\mathbb{R}^{n}$-valued random vector. We denote by $\bar{X}^{\mathrm{f}} \in \mathbb{R}^{n}$ the forecast state mean, $Q^{\mathrm{f}} \in \mathbb{R}^{n \times n}$ the forecast state covariance, and $\bar{X}^{\mathrm{a}} \in \mathbb{R}^{n}$ and $Q^{\mathrm{a}} \in \mathbb{R}^{n \times n}$ the analysis state mean and covariance, respectively. The observation data vector is $d \in \mathbb{R}^{m}$, where $d-H X \sim N(0, R)$, with $H \in \mathbb{R}^{m \times n}$ the linear observation operator, and $R \in \mathbb{R}^{m \times m}, R>0$, is the observation error covariance.

Given the forecast mean and covariance, the Kalman Filter analysis mean and covariance are

$$
\begin{aligned}
& \bar{X}^{\mathrm{a}}=\bar{X}^{\mathrm{f}}+K\left(d-H \bar{X}^{\mathrm{f}}\right) \\
& Q^{\mathrm{a}}=Q^{\mathrm{f}}-Q^{\mathrm{f}} H^{*}\left(H Q^{\mathrm{f}} H^{*}+R\right)^{-1} H Q^{\mathrm{f}}=Q^{\mathrm{f}}-K H Q^{\mathrm{f}},
\end{aligned}
$$

where

$$
K=Q^{\mathrm{f}} H^{*}\left(H Q^{\mathrm{f}} H^{*}+R\right)^{-1}
$$

is the Kalman gain matrix. See, e.g., [1, 16, 31.

In the general case, the state space $\mathbb{R}^{n}$ and the data space $\mathbb{R}^{m}$ above become separable Hilbert spaces, one or both of which may be infinitely dimensional, and matrices become bounded linear operators. The Kalman filter formulas (3.1)-3.3) remain the same. The assumption $R>0$ guarantees that the inverse in (3.3) is well-defined. In particular, when the data space is infinitely dimensional, the definition of a positive definite operator in Section 2.2 implies that this inverse is bounded. In that case, $R$ cannot be the covariance of a probability distribution in the classical sense, because $R>0$ cannot be of trace class in infinite dimension. However, all statistical estimates will be in the state space, not the data space, so this is not a problem.

For future reference, we introduce the operators

$$
\begin{aligned}
\mathcal{K}(Q) & =Q H^{*}\left(H Q H^{*}+R\right)^{-1}, \\
\mathcal{B}(X, Q) & =X+\mathcal{K}(Q)(d-H X), \\
\mathcal{A}(Q) & =Q-Q H^{*}\left(H Q H^{*}+R\right)^{-1} H Q \\
& =Q-\mathcal{K}(Q) H Q,
\end{aligned}
$$

which evaluate the Kalman gain, analysis mean, and the analysis covariance, respectively, in the Kalman filter equations (3.1)-3.3. We are now ready to state the complete Kalman filter for reference. The superscript (k) signifies quantities at time step $k$.

Algorithm 3.1 (Kalman filter). Suppose that the model $\mathcal{M}^{(k)}$ at each time $k \geq 1$ is linear, $\mathcal{M}^{(k)}(X)=M^{(k)} X+b^{(k)}$, and the initial mean $\bar{X}^{(0)}=\bar{X}^{(0), a}$ and the background covariance $B=Q^{(0) \text {,a }}$ of the state are given. At time $k$, the analysis mean and covariance from the previous time $k-1$ are advanced by the model,

$$
\begin{aligned}
\bar{X}^{(k), \mathrm{f}} & =M^{(k)} \bar{X}^{(k-1), \mathrm{a}}+b^{(k)}, \\
Q^{(k), \mathrm{f}} & =M^{(k)} Q^{(k-1), \mathrm{a}} M^{(k)^{*}} .
\end{aligned}
$$


The analysis step incorporates the observation $d^{(k)}$, where $d^{(k)}-H^{(k)} X^{(k), \mathrm{f}}$ has mean zero and covariance $R^{(k)}$, and it gives the analysis mean and covariance

$$
\begin{aligned}
\bar{X}^{(k), \mathrm{a}} & =\mathcal{B}\left(\bar{X}^{(k), \mathrm{f}}, Q^{(k), \mathrm{f}}\right), \\
Q^{(k), \mathrm{a}} & =\mathcal{A}\left(Q^{(k), \mathrm{f}}\right),
\end{aligned}
$$

where $\mathcal{B}$ and $\mathcal{A}$ are defined by (3.5) and (3.7) respectively, with $d, H$, and $R$, at time $k$.

In many applications, the state dimension $n$ of the system is large and computing or even storing the exact covariance of the system state is computationally impractical. Ensemble Kalman filters address this concern. Ensemble Kalman filters use a collection of state vectors, called an ensemble, to represent the distribution of the system state. This ensemble will be denoted by $\boldsymbol{X}_{N}$, comprised of $N$ random elements $X_{i} \in \mathbb{R}^{n}, i=1, \ldots N$. The ensemble mean and ensemble covariance, defined by (2.4) and (2.5), are denoted by $\overline{\boldsymbol{X}}_{N}$ and $Q_{N}$ respectively, while the Kalman Filter mean and covariance are denoted without subscripts, as $\bar{X}$ and $Q$.

There are several ways to produce an analysis ensemble corresponding to the Kalman filter algorithm. Unbiased ensemble square root filters produce an analysis ensemble $\boldsymbol{X}_{N}^{\mathrm{a}}$ such that (3.10) and (3.11) are satisfied for the ensemble mean and covariance. This was not always the case in early variants of ensemble square root filters [30]. Because our results assume a linear model, we formulate the algorithm in the linear case to cut down on additional notation.

Algorithm 3.2 (Unbiased square root ensemble filter). Generate the initial ensemble $\boldsymbol{X}_{N}^{(0), a}$ at time $k=0$ by sampling from a distribution with mean $\bar{X}^{(0)}=\bar{X}^{(0) \text {, a }}$ and covariance $B$. At time $k \geq 1$, the analysis ensemble members $X_{i}^{(k-1) \text {, a }}$ are advanced by the model

$$
X_{i}^{(k), \mathrm{f}}=M^{(k)}\left(X_{i}^{(k-1), \mathrm{a}}\right)+b^{(k)}, \quad i=1, \ldots, N
$$

resulting in the forecast ensemble $\boldsymbol{X}^{(k), \mathrm{f}}$ with ensemble mean and covariance

$$
\overline{\boldsymbol{X}}_{N}^{(k), \mathrm{f}}=E_{N}\left(\boldsymbol{X}_{N}^{(k), \mathrm{f}}\right), \quad Q_{N}^{(k), \mathrm{f}}=C_{N}\left(\boldsymbol{X}_{N}^{(k), \mathrm{f}}\right)
$$

The analysis step creates an ensemble $\overline{\boldsymbol{X}}_{N}^{(k), \text { a }}$ which incorporates the observation that $d^{(k)}-H^{(k)} \overline{\boldsymbol{X}}_{N}^{(k), \mathrm{f}}$ has mean zero and covariance $R^{(k)}$. The analysis ensemble is constructed (in a manner determined by the specific method) to have the ensemble mean and covariance given by

$$
\begin{aligned}
& E_{N}\left(\overline{\boldsymbol{X}}_{N}^{(k), \mathrm{a}}\right)=\mathcal{B}\left(\overline{\boldsymbol{X}}_{N}^{(k), \mathrm{f}}, Q_{N}^{(k), \mathrm{f}}\right)=\overline{\boldsymbol{X}}_{N}^{(k), \mathrm{f}}+\mathcal{K}\left(Q_{N}^{(k), \mathrm{f}}\right)\left(d^{(k)}-H\left(\overline{\boldsymbol{X}}_{N}^{(k), \mathrm{f}}\right)\right), \\
& C_{N}\left(\overline{\boldsymbol{X}}_{N}^{(k), \mathrm{a}}\right)=\mathcal{A}\left(Q_{N}^{(k), \mathrm{f}}\right)=Q_{N}^{(k), \mathrm{f}}-\mathcal{K}\left(Q_{N}^{(k), \mathrm{f}}\right) H^{(k)} Q_{N}^{(k), \mathrm{f}},
\end{aligned}
$$

where $\mathcal{B}$ and $\mathcal{A}$ are defined by (3.5) and (3.7) with $d, H$, and $R$, at time $k$.

The Ensemble Adjustment Kalman Filter (EAKF, 2]), the filter by Whitaker and Hamill [34, and the Ensemble Transform Kalman Filter (ETKF, 6]) and its variants, the Local Ensemble Transform Kalman Filter (LETKF, 15]) and its revision [33, satisfy properties (3.14) and (3.15), cf., [23, 32, and therefore are of this form.

The background covariance $B$ does not need to be stored explicitly. Rather, it is used in a factored form [7. 13 ,

$$
B=L L^{\mathrm{T}}
$$

and only multiplication of $L$ times a vector is needed,

$$
X_{i}^{(0)}=X^{(0)}+L Y_{i}, \quad Y_{i} \sim N(0, I)
$$

For example, a sample covariance of the form (3.16) can be created from historical data. To better represent the dominant part of the background covariance, $L$ can consist of approximate eigenvectors for the largest eigenvalues, obtained by a variant of the power method [17. Another popular choice is $L=T S$, 
where $T$ is a transformation, such as FFT or wavelet transform, which requires no storage of the matrix entries at all, and $S$ is a sparse matrix [11, 27, 29. The covariance of the Fourier transform of a stationary random field is diagonal, so even an approximation with diagonal matrix $S$ is useful, and computationally very inexpensive.

A key observation of our analysis is that, in the linear case, the transformations of the ensemble mean and covariance in unbiased square root ensemble filters are exactly the same as in the Kalman filter, and they can be described without reference to the ensemble at all, as shown in the next lemma. All that is needed for the convergence of the unbiased square root ensemble filter is the convergence of the initial ensemble mean and covariance, and the continuity of the transformations, in a suitable statistical sense.

LEMma 3.3. The transformations of the mean and covariance in step $k$ of Algorithms 3.1] and [3.2 are the same,

$$
\begin{array}{c|c}
\text { Kalman Filter } & \begin{array}{c}
\text { Unbiased Square Root } \\
\text { Ensemble Kalman Filter }
\end{array} \\
\bar{X}^{(k), \mathrm{f}}=M^{(k)} \bar{X}^{(k-1), \mathrm{a}}+b^{(k)} & \overline{\boldsymbol{X}}_{N, \mathrm{f}}^{(k)}=M^{(k)} \overline{\boldsymbol{X}}_{N}^{(k-1), \mathrm{a}}+b^{(k)} \\
Q^{(k), \mathrm{f}}=M^{(k)} Q^{(k-1), \mathrm{a}} M^{(k) *} & Q_{N}^{(k), \mathrm{f}}=M^{(k)} Q_{N}^{(k-1), \mathrm{a}} M^{(k) *} \\
\bar{X}^{(k), \mathrm{a}}=\mathcal{B}\left(\bar{X}^{(k), \mathrm{f}}, Q^{(k), \mathrm{f}}\right) & \overline{\boldsymbol{X}}_{N}^{(k), \mathrm{a}}=\mathcal{B}\left(\overline{\boldsymbol{X}}_{N}^{(k), \mathrm{f}}, Q_{N}^{(k), \mathrm{f}}\right) \\
Q^{(k), \mathrm{a}}=\mathcal{A}\left(Q^{(k), \mathrm{f}}\right) & Q_{N}^{(k), \mathrm{a}}=\mathcal{A}\left(Q_{N}^{(k), \mathrm{f}}\right)
\end{array}
$$

Proof. From (3.13) and the definition (2.5) of ensemble covariance, it follows that

$$
Q_{N}^{(k), \mathrm{f}}=M^{(k)} Q_{N}^{(k-1), \mathrm{a}} M^{(k)^{*}} .
$$

The rest of the transformations in Algorithms 3.1 and 3.2 are already the same.

4. Continuity of the analysis step. Fundamental to our analysis are continuity estimates for the operators $\mathcal{A}$ and $\mathcal{B}$, which bring the forecast statistics to the analysis statistics. Our general strategy is to first derive pointwise estimates which apply to every realization of the random elements separately, then integrate them to get the corresponding $L^{p}$ estimates. We will prove the following estimates for general covariances $Q$ and $P$, with the state covariance and sample covariance of the filters in mind. Likewise, estimates will be made with general elements $X$ and $Y$, with the state mean and sample mean of the filters in mind.

4.1. Pointwise bounds. The first estimate is the continuity of the Kalman gain matrix (or operator) (3.3) as a function of the forecast covariance, shown in the next lemma and its corollary. See also [22, Proposition 22.2].

Lemma 4.1. If $R>0$ and $P, Q \geq 0$, then

$$
|\mathcal{K}(Q)-\mathcal{K}(P)| \leq|Q-P||H|\left|R^{-1}\right|\left(1+\min \{|P|,|Q|\}|H|^{2}\left|R^{-1}\right|\right) .
$$

Proof. Since $R>0$ and $P, Q \geq 0, \mathcal{K}(Q)$ and $\mathcal{K}(P)$ are defined in (3.4). For $A, B \geq 0$, we have the identity,

$$
(I+A)^{-1}-(I+B)^{-1}=(I+A)^{-1}(B-A)(I+B)^{-1},
$$

which is verified by multiplication by $I+A$ on the left and $I+B$ on the right, and

$$
\left|(I+A)^{-1}-(I+B)^{-1}\right| \leq|B-A|,
$$

which follows from (4.1) using the inequalities $\left|(I+A)^{-1}\right| \leq 1,\left|(I+B)^{-1}\right| \leq 1$, because $A, B \geq 0$. Now write

$$
\left(H Q H^{*}+R\right)^{-1}=R^{-1 / 2}\left(R^{-1 / 2} H Q H^{*} R^{-1 / 2}+I\right)^{-1} R^{-1 / 2},
$$


using the symmetric square root $R^{1 / 2}$ of $R$. By (4.2) with $A=R^{-1 / 2} H Q H^{*} R^{-1 / 2}$ and $B=$ $R^{-1 / 2} H P H^{*} R^{-1 / 2}$ and (4.3), we have that

$$
\left|\left(H Q H^{*}+R\right)^{-1}-\left(H P H^{*}+R\right)^{-1}\right| \leq|Q-P||H|^{2}\left|R^{-1}\right|^{2} .
$$

Since $H Q H^{*}+R \geq R$, we have

$$
\left|\left(H Q H^{*}+R\right)^{-1}\right| \leq\left|R^{-1}\right|
$$

Using (4.4), (4.5), and the definition of the operator $\mathcal{K}$ from (3.4), we have

$$
\begin{aligned}
|\mathcal{K}(Q)-\mathcal{K}(P)|= & \left|Q H^{*}\left(H Q H^{*}+R\right)^{-1}-P H^{*}\left(H P H^{*}+R\right)^{-1}\right| \\
= & \mid Q H^{*}\left(H Q H^{*}+R\right)^{-1}-P H^{*}\left(H Q H^{*}+R\right)^{-1} \\
& +P H^{*}\left(H Q H^{*}+R\right)^{-1}-P H^{*}\left(H P H^{*}+R\right)^{-1} \mid \\
\leq & |Q-P||H|\left|R^{-1}\right|\left(1+|P||H|^{2}\left|R^{-1}\right|\right) .
\end{aligned}
$$

Swapping the roles $P$ and $Q$ yields

$$
|\mathcal{K}(Q)-\mathcal{K}(P)| \leq|Q-P||H|\left|R^{-1}\right|\left(1+|Q||H|^{2}\left|R^{-1}\right|\right),
$$

which completes the proof.

A pointwise bound on the Kalman gain follows.

Corollary 4.2. If $R>0$ and $Q \geq 0$, then

$$
|\mathcal{K}(Q)| \leq|Q||H|\left|R^{-1}\right| .
$$

Proof. Use Lemma 4.1 with $P=0$.

Corollary 4.3. If $R>0$ and $Q \geq 0$, then

$$
|\mathcal{B}(X, Q)| \leq|X|+\left|Q\|H\| R^{-1}\right|(|d-H X|) .
$$

Proof. By the definition of operator $\mathcal{B}$ in (3.5), the pointwise bound on the Kalman gain (4.6), and the triangle inequality,

$$
|\mathcal{B}(X, Q)|=|X+\mathcal{K}(Q)(d-H X)| \leq|X|+|Q||H|\left|R^{-1}\right|(|d-H X|) .
$$

The pointwise continuity of operator $\mathcal{A}$ also follows from Lemma 4.1. To reduce the notation we introduce the constant

$$
c=|H|^{2}\left|R^{-1}\right|
$$

LEMMA 4.4. If $R>0$ and $P, Q \geq 0$, then

$$
|\mathcal{A}(Q)-\mathcal{A}(P)| \leq|Q-P|\left(1+c|Q|+c|P|+c^{2}|P||Q|\right) .
$$

Proof. Since $R>0$ and $P, Q \geq 0$, it follows that $\mathcal{K}(Q), \mathcal{K}(P), \mathcal{A}(Q)$, and $\mathcal{A}(P)$ are defined. From the definition of operator $\mathcal{A}$ (3.7), Lemma 4.1, and Corollary 4.2,

$$
\begin{aligned}
|\mathcal{A}(Q)-\mathcal{A}(P)| & =|(Q-\mathcal{K}(Q) H Q)-(P-\mathcal{K}(P) H P)| \\
& =|Q-P+\mathcal{K}(P) H P-\mathcal{K}(Q) H Q| \\
& =|Q-P+\mathcal{K}(P) H P-\mathcal{K}(P) H Q+\mathcal{K}(P) H Q-\mathcal{K}(Q) H Q| \\
& \leq|Q-P|\left(1+c|Q|+c|P|+c^{2}|P||Q| \mid\right) . \quad
\end{aligned}
$$


REMARK 4.5. The choice of $|P|$ in $\min \{|P|,|Q|\}$ in the proof of Lemma 4.4 was made to preserve symmetry. Swapping the roles of $P$ and $Q$ in the proof gives a sharper, but a more complicated bound

$$
|\mathcal{A}(Q)-\mathcal{A}(P)| \leq|Q-P|\left(1+c|Q|+c|P|+c^{2} \cdot \min \left\{|P|^{2},|P||Q|,|Q|^{2}\right\}\right) .
$$

Instead of setting $P=0$ above, we can get a well-known sharper pointwise bound on $\mathcal{A}(Q)$ directly from (3.6). The proof is written in a way suitable for our generalization.

Lemma 4.6. If $R>0$ and $Q \geq 0$, then

$$
0 \leq \mathcal{A}(Q) \leq Q
$$

and

$$
|\mathcal{A}(Q)| \leq|Q|
$$

Proof. By the definition of operator $\mathcal{A}$ in (3.6),

$$
\mathcal{A}(Q)=Q-Q H^{*}\left(H Q H^{*}+R\right)^{-1} H Q \leq Q,
$$

because $Q H^{*}\left(H Q H^{*}+R\right)^{-1} H Q \geq 0$. It remains to show that $\mathcal{A}(Q) \geq 0$. Note that for any $A$, since $R>0$,

$$
A^{*} A+R \geq A^{*} A,
$$

and, consequently,

$$
\left(A^{*} A+R\right)^{-1 / 2} A^{*} A\left(A^{*} A+R\right)^{-1 / 2} \leq I .
$$

Since for $B \geq 0, B \leq I$ is the same as the spectral radius $\rho(B) \leq 1$, and, for any $C$ and $D, \rho(C D)=\rho(D C)$, we have

$$
A\left(A^{*} A+R\right)^{-1} A^{*} \leq I
$$

Using (4.11) with $A=Q^{1 / 2} H^{*}$ gives

$$
Q^{1 / 2} H^{*}\left(H Q H^{*}+R\right)^{-1} H Q^{1 / 2} \leq I,
$$

and, consequently

$$
Q H^{*}\left(H Q H^{*}+R\right)^{-1} H Q \leq Q
$$

which gives $\mathcal{A}(Q) \geq 0$.

Since $\mathcal{A}(Q)$ and $Q$ are symmetric, (4.9) implies (4.10).

The pointwise continuity of operator $\mathcal{B}$ follows from Lemma 4.1 as well.

Lemma 4.7. If $R>0$, and $P, Q \geq 0$, then,

$$
|\mathcal{B}(X, Q)-\mathcal{B}(Y, P)| \leq|X-Y|(1+c|Q|)+|Q-P||H|\left|R^{-1}\right|(1+c|P|)(|d-H Y|) .
$$

Proof. Estimating the difference, and using the pointwise bound on the Kalman gain (4.6) and the pointwise continuity of the Kalman gain from Lemma 4.1 .

$$
\begin{aligned}
\mid \mathcal{B}(X, Q) & -\mathcal{B}(Y, P) \mid \\
& =|(X+\mathcal{K}(Q)(d-H X))-(Y+\mathcal{K}(P)(d-H Y))| \\
& =|X-Y+\mathcal{K}(Q)(H Y-H X)+(\mathcal{K}(Q)-\mathcal{K}(P))(d-H Y)| \\
& \leq|X-Y|+c|Q||X-Y|+|Q-P||H|\left|R^{-1}\right|(1+c|P|)(|d-H Y|)
\end{aligned}
$$

which is (4.12). 
4.2. $L^{p}$ bounds. Using the pointwise estimate on the continuity of operator $\mathcal{A}$, we can now estimate its continuity in $L^{p}$ spaces of random vectors. We will only need the result with one of the arguments random and the other one constant (i.e., non random), which simplifies its statement and proof. This is because the application of these estimates will be the ensemble sample covariance, which is random, and the state covariance, which is constant. In a similar way we will estimate the continuity of operator $\mathcal{B}$ in $L^{p}$.

Lemma 4.8. Let $Q$ be a random operator such that $Q \geq 0$ almost surely (a.s.), let $P \geq 0$ be constant, and let $R>0$. Then, for all $1 \leq p<\infty$,

$$
\|\mathcal{A}(Q)-\mathcal{A}(P)\|_{p} \leq(1+c|P|)\|Q-P\|_{p}+\left(c+c^{2}|P|\right)\|Q\|_{2 p}\|Q-P\|_{2 p} .
$$

Proof. From Lemma 4.4, the triangle inequality, Lemma 2.1, and recognizing that $P$ is constant,

$$
\begin{aligned}
\|\mathcal{A}(Q)-\mathcal{A}(P)\|_{p} \leq & \left\||Q-P|\left(1+c|Q|+c|P|+c^{2}|P \| Q|\right)\right\|_{p} \\
\leq & \|Q-P\|_{p}+c\|Q\|_{2 p}\|Q-P\|_{2 p} \\
& \quad+c|P|\|Q-P\|_{p}+c^{2}|P|\|Q\|_{2 p}\|Q-P\|_{2 p} \\
= & (1+c|P|)\|Q-P\|_{p}+\left(c+c^{2}|P|\right)\|Q\|_{2 p}\|Q-P\|_{2 p} .
\end{aligned}
$$

Instead of setting $P=0$ above, we get a better bound on $\|\mathcal{A}(Q)\|_{p}$ directly.

Lemma 4.9. Let $Q$ be a random operator such that $Q \geq 0$ a.s., and $R>0$. Then, for all $1 \leq p<\infty$,

$$
\|\mathcal{A}(Q)\|_{p} \leq\|Q\|_{p} .
$$

Proof. From (4.10), it follows that $E\left(|\mathcal{A}(Q)|^{p}\right) \leq E\left(|Q|^{p}\right)$.

Using the pointwise estimate on the continuity of the operator $\mathcal{B}$, we estimate its continuity in $L^{p}$ spaces of random vectors. Again, we keep the arguments of one of the terms constant, which is all we will need, resulting in a simplification.

Lemma 4.10. Let $Q$ and $X$ be random operators, and $P$ and $Y$ be constant. Let $Q \geq 0$ a.s., $P \geq 0$, and $R>0$. Then, for all $1 \leq p<\infty$,

$$
\begin{aligned}
\|\mathcal{B}(X, Q)-\mathcal{B}(Y, P)\|_{p} \leq \| & X-Y\left\|_{p}+c\right\| Q\left\|_{2 p}\right\| X-Y \|_{2 p} \\
& +\|Q-P\|_{p}\left|H \| R^{-1}\right|(1+c|P|)(|d-H Y|) .
\end{aligned}
$$

Proof. Applying the $L^{p}$ norm to the pointwise bound (4.12), using the triangle inequality, recognizing that $P$ and $Y$ are constant, and applying the Cauchy-Schwarz inequality (Lemma 2.1), we get

$$
\begin{aligned}
\|\mathcal{B}(X, Q)-\mathcal{B}(Y, P)\|_{p} & =\left\||X-Y|(1+c|Q|)+\left|Q-P\|H\| R^{-1}\right|(1+c|P|)|d-H Y|\right\|_{p} \\
\leq & \|X-Y\|_{p}+c\|Q\|_{2 p}\|X-Y\|_{2 p} \\
& +\|Q-P\|_{p}\left|H \| R^{-1}\right|(1+c|P|)(|d-H Y|) .
\end{aligned}
$$

5. $L^{p}$ laws of large numbers. Similarly as in [22, 24, we will work with convergence in all $L^{p}$, $1 \leq p<\infty$. To prove $L^{p}$ convergence of the initial ensemble mean and covariance to the mean and covariance of the background distribution, we need the corresponding laws of large numbers.

5.1. $L^{p}$ convergence of the sample mean. The $L^{2}$ law of large numbers for $X_{1}, \ldots, X_{N} \in L^{2}(V)$ i.i.d. is classical:

$$
\left\|E_{N}\left(\boldsymbol{X}_{N}\right)-E\left(X_{1}\right)\right\|_{2} \leq \frac{1}{\sqrt{N}}\left\|X_{1}-E\left(X_{1}\right)\right\|_{2} \leq \frac{2}{\sqrt{N}}\left\|X_{1}\right\|_{2} .
$$

The proof relies on the expansion (assuming $E\left(X_{1}\right)=0$ without loss of generality),

$$
\begin{aligned}
\left\|E_{N}\left(\boldsymbol{X}_{N}\right)\right\|_{2}^{2} & =E\left(\left\langle\frac{1}{N} \sum_{i=1}^{N} X_{i}, \frac{1}{N} \sum_{i=1}^{N} X_{i}\right\rangle\right)=\frac{1}{N^{2}} \sum_{i=1}^{N} \sum_{j=1}^{N} E\left(\left\langle X_{i}, X_{j}\right\rangle\right) \\
& =\frac{1}{N^{2}} \sum_{i=1}^{N} E\left(\left\langle X_{i}, X_{i}\right\rangle\right)=\frac{1}{N} E\left(\left\langle X_{1}, X_{1}\right\rangle\right)=\frac{1}{N}\left\|X_{1}\right\|_{2}^{2},
\end{aligned}
$$


which yields $\left\|E_{N}\left(\boldsymbol{X}_{N}\right)\right\|_{2} \leq\left\|X_{1}\right\|_{2} / \sqrt{N}$. To obtain $L^{p}$ laws of large numbers for $p \neq 2$, the equality (5.2) needs to be replaced by the following.

Lemma 5.1. (Marcinkiewicz-Zygmund inequality) If $1 \leq p<\infty$ and $Y_{i} \in L^{p}(V), E\left(Y_{i}\right)=0$ and $E\left(\left|Y_{i}\right|^{p}\right)<\infty, i=1, \ldots, N$, then

$$
E\left(\left|\sum_{i=1}^{N} Y_{i}\right|^{p}\right) \leq B_{p} E\left(\sum_{i=1}^{N}\left|Y_{i}\right|^{2}\right)^{p / 2},
$$

where $B_{p}$ depends on $p$ only.

Proof. For the finite dimensional case, see [26] or [9, p. 367]. In the infinitely dimensional case, note that a separable Hilbert space is a Banach space of Rademacher type 2 [5, p. 159], which implies (5.3) for any $p \geq 1$ [35, Proposition 2.1]. All infinitely dimensional separable Hilbert spaces are isometric, so the same $B_{p}$ works for any of them.

The Marcinkiewicz-Zygmund inequality begets a variant of the weak law of large numbers in $L^{p}$ norms, similarly as in [9, Corollary 2, page 368]. Note that the Marcinkiewicz-Zygmund inequality does not hold in general Banach spaces, and in fact it characterizes Banach spaces of Rademacher type 2 [35, Proposition 2.1], so it is important that $V=\mathbb{R}^{n}$ or $V$ is a separable Hilbert space, as assumed throughout.

Theorem 5.2. Let $2 \leq p<\infty$ and $X_{1}, \ldots, X_{N} \in L^{p}(V)$ be i.i.d. Then,

$$
\left\|E_{N}\left(\boldsymbol{X}_{N}\right)-E\left(X_{1}\right)\right\|_{p} \leq \frac{C_{p}}{\sqrt{N}}\left\|X_{1}-E\left(X_{1}\right)\right\|_{p} \leq \frac{2 C_{p}}{\sqrt{N}}\left\|X_{1}\right\|_{p},
$$

where $C_{p}$ depends on $p$ only.

Proof. If $p=2$, the statement becomes (5.1). Let $p>2$, and consider the case $E\left(X_{1}\right)=0$. By Hölder's inequality with the conjugate exponents $\frac{p-2}{p}$ and $\frac{2}{p}$,

$$
\begin{aligned}
\sum_{i=1}^{N}\left|X_{i}\right|^{2} & =\sum_{i=1}^{N} 1\left(\left|X_{i}\right|^{2}\right) \leq\left(\sum_{i=1}^{N}\left(1^{p / p-2}\right)\right)^{(p-2) / p}\left(\sum_{i=1}^{N}\left(\left|X_{i}\right|^{2}\right)^{p / 2}\right)^{2 / p} \\
& =N^{(p-2) / p}\left(\sum_{i=1}^{N}\left|X_{i}\right|^{p}\right)^{2 / p}
\end{aligned}
$$

Using the Marcinkiewicz-Zygmund inequality (5.3) and (5.5),

$$
\begin{aligned}
E\left(\left|\sum_{i=1}^{N} X_{i}\right|^{p}\right) & \leq B_{p} E\left(\left(\sum_{i=1}^{N}\left|X_{i}\right|^{2}\right)^{p / 2}\right) \\
& \leq B_{p} E\left(\left(N^{(p-2) / p}\left(\sum_{i=1}^{N}\left|X_{i}\right|^{p}\right)^{2 / p}\right)^{p / 2}\right) \\
& \leq B_{p} N^{p / 2-1} E\left(\sum_{i=1}^{N}\left|X_{i}\right|^{p}\right)=B_{p} N^{p / 2} E\left(\left|X_{1}\right|^{p}\right),
\end{aligned}
$$

because $\frac{p}{2} \frac{p-2}{p}=\frac{p}{2}-1$, and the $X_{i}$ are identically distributed. Taking the $p$-th root of both sides of (5.6) yields

$$
\left\|\sum_{i=1}^{N} X_{i}\right\|_{p} \leq B_{p}^{1 / p} N^{1 / 2}\left\|X_{1}\right\|_{p},
$$

and the first inequality in (5.4) follows after dividing by $N$. The general case when $E\left(X_{1}\right) \neq 0$ follows from the triangle inequality. 
5.2. $L^{p}$ convergence of the sample covariance. For the convergence of the ensemble covariance, we use the $L^{p}$ law of large numbers in Hilbert-Schmidt norm, because HS $(V)$ is a separable Hilbert space, while $[V]$ is not even a Hilbert space. Convergence in the norm of $[V]$, the operator norm, then follows from (2.1). See also [22, Lemma 22.3] for a related result using entry-by-entry estimates.

Theorem 5.3. Let $X_{1}, \ldots, X_{N} \in L^{2 p}(V)$ be i.i.d. and $p \geq 2$. Then

$$
\left\|\left|C_{N}\left(\boldsymbol{X}_{N}\right)-\operatorname{Cov}\left(X_{1}\right)\right|_{\mathrm{HS}}\right\|_{p} \leq\left(\frac{2 C_{p}}{\sqrt{N}}+\frac{4 C_{2 p}^{2}}{N}\right)\left\|X_{1}\right\|_{2 p}^{2} .
$$

where $C_{p}$ is a constant which depends on $p$ only; in particular, $C_{2}=1$.

Proof. Without loss of generality, let $E\left(X_{1}\right)=0$. Then

$$
\begin{aligned}
C_{N}\left(\boldsymbol{X}_{N}\right) & -\operatorname{Cov}\left(X_{1}\right) \\
& =\left(E_{N}\left(\boldsymbol{X}_{N} \otimes \boldsymbol{X}_{N}\right)-E\left(X_{1} \otimes X_{1}\right)\right)-E_{N}\left(\boldsymbol{X}_{N}\right) \otimes E_{N}\left(\boldsymbol{X}_{N}\right),
\end{aligned}
$$

where $\boldsymbol{X}_{N} \otimes \boldsymbol{X}_{N}=X_{1} \otimes X_{1}+\ldots+X_{N} \otimes X_{N}$. For the first term in (5.8), the $L^{p}$ law of large numbers (5.4) in $\operatorname{HS}(V)$ yields

$$
\begin{aligned}
E\left(\left|E_{N}\left(\boldsymbol{X}_{N} \otimes \boldsymbol{X}_{N}\right)-E\left(X_{1} \otimes X_{1}\right)\right|_{\mathrm{HS}}^{p}\right)^{1 / p} & \leq \frac{2 C_{p}}{\sqrt{N}} E\left(\left|X_{1} \otimes X_{1}\right|_{\mathrm{HS}}^{p}\right)^{1 / p} \\
& =\frac{2 C_{p}}{\sqrt{N}} E\left(\left|X_{1}\right|^{2 p}\right)^{1 / p}=\frac{2 C_{p}}{\sqrt{N}}\left\|X_{1}\right\|_{2 p}^{2},
\end{aligned}
$$

using (2.2). For the second term in (5.8), we use again (2.2) to get

$$
\left|E_{N}\left(\boldsymbol{X}_{N}\right) \otimes E_{N}\left(\boldsymbol{X}_{N}\right)\right|_{\mathrm{HS}}=\left|E_{N}\left(\mathbf{X}_{N}\right)\right|^{2}
$$

and the $L^{p}$ law of large numbers in $V$ yields

$$
\begin{aligned}
E\left(\left|E_{N}\left(\boldsymbol{X}_{N}\right) \otimes E_{N}\left(\boldsymbol{X}_{N}\right)\right|_{H S}^{p}\right)^{1 / p} & =E\left(\left|E_{N}\left(\boldsymbol{X}_{N}\right)\right|^{2 p}\right)^{1 / p} \\
& =\left\|E_{N}\left(\boldsymbol{X}_{N}\right)-0\right\|_{2 p}^{2} \leq\left(\frac{2 C_{2 p}}{\sqrt{N}}\left\|X_{1}\right\|_{2 p}\right)^{2} .
\end{aligned}
$$

It remains to use the triangle inequality for the general case.

6. Convergence of the unbiased squared root filter. By the law of large numbers, the sample mean and the sample covariance of the initial ensemble converge to the mean and covariance of the background distribution. Every analysis step is a continuous mapping of the mean and covariance, and the convergence in the large ensemble limit follows. The theorem below, which is the main result of this paper, quantifies this argument.

THeOREM 6.1. Assume that the state space $V$ is a finite dimensional or separable Hilbert space, the initial state, denoted $X^{(0)}$, has a distribution on $V$ such that all moments exist, $E\left(\left|X^{(0)}\right|^{p}\right)<\infty$ for every $1 \leq p<\infty$, the initial ensemble $\boldsymbol{X}_{N}^{(0)}$ is an i.i.d. sample from this distribution, and the model is linear, $\mathcal{M}^{(k)}(X)=M^{(k)} X+b^{(k)}$ for every time $k$.

Then, for all $k \geq 0$, the ensemble mean $\overline{\boldsymbol{X}}_{N}^{(k), \mathrm{a}}$ and covariance $Q_{N}^{(k), \mathrm{a}}$ from the unbiased square root ensemble filter (Algorithm 3.2) converge to the mean $\bar{X}^{(k), a}$ and covariance $Q^{(k) \text {,a }}$ from the Kalman filter (Algorithm [3.1), respectively, in all $L^{p}, 1 \leq p<\infty$, as $N \rightarrow \infty$, with the convergence rate $1 / \sqrt{N}$. Specifically,

$$
\begin{gathered}
\left\|\overline{\boldsymbol{X}}_{N}^{(k), \mathrm{a}}-\bar{X}^{(k), \mathrm{a}}\right\|_{p} \leq \frac{\operatorname{const}(p, k)}{\sqrt{N}}, \\
\left\|Q_{N}^{(k), \mathrm{a}}-Q^{(k), \mathrm{a}}\right\|_{p} \leq \frac{\operatorname{const}(p, k)}{\sqrt{N}}
\end{gathered}
$$


for all $1 \leq p<\infty$, and all $N=1,2, \ldots$, where we use const $(p, k)$ to denote a generic constant which depends on $p, k$, on the norms of the various constant (non-random) inputs and operators in the problem, and on the background distribution, but not on the dimension of the state space or the ensemble size.

Proof. We will proceed by induction on $k$. For $k=0$ and $p \geq 2$, (6.1) and (6.2) follow immediately from the $L^{p}$ laws of large numbers, (5.4) and (5.7), respectively. For $1 \leq p<2$, it is sufficient to note that the $L^{p}$ norm is dominated by the $L^{2}$ norm.

Let $k \geq 1$ and assume that (6.1) and (6.2) hold with $k-1$ in place of $k$, for all $N>0$, and all $1 \leq p<\infty$. From (3.8), (3.9), and (3.17), it follows that

$$
\left|\bar{X}^{(k), \mathrm{f}}\right|,\left|Q^{(k), \mathrm{f}}\right| \leq \operatorname{const}(k), \quad\left\|Q_{N}^{(k), \mathrm{f}}\right\|_{p} \leq \operatorname{const}(p, k),
$$

for all $1 \leq p<\infty$. Comparing (3.9) and (3.17), we have

$$
\begin{aligned}
\left\|Q_{N}^{(k), \mathrm{f}}-Q^{(k), \mathrm{f}}\right\|_{p} & =\left\|M^{(k)} Q_{N}^{(k-1), \mathrm{a}} M^{(k) *}-M^{(k)} Q^{(k-1), \mathrm{a}} M^{(k) *}\right\|_{p} \\
& \leq\left|M^{(k)}\right|^{2}\left\|Q_{N}^{(k-1), \mathrm{a}}-Q^{(k-1), \mathrm{a}}\right\|_{p},
\end{aligned}
$$

and from Lemma 4.8 ,

$$
\begin{aligned}
& \left\|Q_{N}^{(k), \mathrm{a}}-Q^{(k), \mathrm{a}}\right\|_{p}=\left\|\mathcal{A}\left(Q_{N}^{(k), \mathrm{f}}\right)-\mathcal{A}\left(Q^{(k), \mathrm{a}}\right)\right\|_{p} \\
& \leq\left(1+c^{(k)}\left|Q^{(k), \mathrm{f}}\right|\right)\left\|Q_{N}^{(k), \mathrm{f}}-Q^{(k), \mathrm{f}}\right\|_{p} \\
& \left.\quad+\left(c^{(k)}+\left(c^{(k)}\right)^{2}\right)\left|Q^{(k), \mathrm{f}}\right|\right)\left\|Q_{N}^{(k), \mathrm{f}}\right\|_{2 p}\left\|Q_{N}^{(k), \mathrm{f}}-Q^{(k), \mathrm{f}}\right\|_{2 p},
\end{aligned}
$$

where $c^{(k)}=\left|H^{(k)}\right|^{2}\left|\left(R^{(k)}\right)^{-1}\right|$. Combining (6.3)-(6.5) and using the induction assumption (6.2), with $2 p$ in place of $p$ when necessary, we obtain

$$
\left\|Q_{N}^{(k), \mathrm{a}}-Q^{(k), \mathrm{a}}\right\|_{p} \leq \frac{\operatorname{const}(p, k)}{\sqrt{N}} .
$$

For the convergence of the ensemble mean to the Kalman filter mean, we have

$$
\left\|\overline{\boldsymbol{X}}_{N}^{(k), \mathrm{f}}-\bar{X}^{(k), \mathrm{f}}\right\|_{p}=\left\|M^{(k)}\left(\overline{\boldsymbol{X}}_{N}^{(k-1), \mathrm{a}}-\bar{X}^{(k-1), \mathrm{a}}\right)\right\|_{p} \leq\left|M^{(k)}\right|\left\|\overline{\boldsymbol{X}}_{N}^{(k-1), \mathrm{a}}-\bar{X}^{(k-1), \mathrm{a}}\right\|_{p},
$$

from Lemma 3.3. By Lemma 4.10 .

$$
\begin{aligned}
\left\|\overline{\boldsymbol{X}}_{N}^{(k), \mathrm{a}}-\bar{X}^{(k), \mathrm{a}}\right\|_{p} & =\left\|\mathcal{B}\left(\overline{\boldsymbol{X}}_{N}^{(k), \mathrm{f}}, Q_{N}^{(k), \mathrm{f}}\right)-\mathcal{B}\left(\bar{X}^{(k), \mathrm{f}}, Q^{(k), \mathrm{f}}\right)\right\|_{p} \\
\leq & \left\|\overline{\boldsymbol{X}}_{N}^{(k), \mathrm{f}}-\bar{X}^{(k), \mathrm{f}}\right\|_{p}+c^{(k)}\left\|Q_{N}^{(k), \mathrm{f}}\right\|_{2 p}\left\|\overline{\boldsymbol{X}}_{N}^{(k), \mathrm{f}}-\bar{X}^{(k), \mathrm{f}}\right\|_{2 p} \\
& +\left\|Q_{N}^{(k), \mathrm{f}}-Q^{(k), \mathrm{f}}\right\|_{p}\left|H \| R^{-1}\right|\left(1+c^{(k)}\left|Q^{(k), \mathrm{f}}\right|\right)\left(\left|d-H \bar{X}^{(k), \mathrm{f}}\right|\right),
\end{aligned}
$$

which, together with (6.3) and (6.4), and the induction assumption (6.2), with $2 p$ in place of $p$ when necessary, yields

$$
\left\|\overline{\boldsymbol{X}}_{N}^{(k), \mathrm{a}}-\bar{X}^{(k), \mathrm{a}}\right\|_{p} \leq \frac{\text { const }(p, k)}{\sqrt{N}}
$$

\section{REFERENCES}

[1] Brian D. O. Anderson and John B. Moore, Optimal filtering, Prentice-Hall, Englewood Cliffs, N.J., 1979.

[2] Jeffrey L. Anderson, An ensemble adjustment Kalman filter for data assimilation, Monthly Weather Review, 129 (2001), pp. 2884-2903.

[3] J. L. ANDERSON, An adaptive covariance inflation error correction algorithm for ensemble filters, Tellus A, 59 (2007), pp. 210-224.

[4] Jeffrey L. Anderson and Stephen L. Anderson, A Monte Carlo implementation of the nonlinear filtering problem to produce ensemble assimilations and forecasts, Monthly Weather Review, 127 (1999), pp. 2741-2758. 
[5] Aloisio Araujo and Evarist Giné, The central limit theorem for real and Banach valued random variables, Wiley Series in Probability and Mathematical Statistics, John Wiley \& Sons, New York-Chichester-Brisbane, 1980.

[6] Craig H. Bishop, Brian J. Etherton, and Sharanya J. Majumdar, Adaptive sampling with the ensemble transform Kalman filter. Part I: Theoretical aspects, Monthly Weather Review, 129 (2001), pp. 420-436.

[7] Mark Buehner, Ensemble-derived stationary and flow-dependent background-error covariances: Evaluation in a quasioperational NWP setting, Quarterly Journal of the Royal Meteorological Society, 131 (2005), pp. 1013-1043.

[8] Gerrit Burgers, Peter Jan van Leeuwen, and Geir Evensen, Analysis scheme in the ensemble Kalman filter, Monthly Weather Review, 126 (1998), pp. 1719-1724.

[9] Yuan Shin Chow and Henry Teicher, Probability theory. Independence, interchangeability, martingales, SpringerVerlag, New York, second ed., 1988.

[10] Giuseppe Da Prato, An introduction to infinite-dimensional analysis, Springer-Verlag, Berlin, 2006.

[11] Alex Deckmyn and Lö̈K Berre, A wavelet approach to representing background error covariances in a limited-area model, Monthly Weather Review, 133 (2005), pp. 1279-1294.

[12] Geir Evensen, Data Assimilation: The Ensemble Kalman Filter, Springer, 2nd ed., 2009.

[13] M. Fisher AND P. Courtier, Estimating the covariance matrices of analysis and forecast error in variational data assimilation. ECMWF Research Department Tech. Memo 220, 1995.

[14] Reinhard Furrer and Thomas Bengtsson, Estimation of high-dimensional prior and posterior covariance matrices in Kalman filter variants, J. Multivariate Anal., 98 (2007), pp. 227-255.

[15] B. R. Hunt, E. J. Kostelich, and I. Szunyogh, Efficient data assimilation for spatiotemporal chaos: a local ensemble transform Kalman filter, Physica D: Nonlinear Phenomena, 230 (2007), pp. 112-126.

[16] Andrew H. Jazwinski, Stochastic processes and filtering theory, Academic Press, New York, 1970.

[17] Eugenia Kalnay, Atmospheric Modeling, Data Assimilation and Predictability, Cambridge University Press, 2003.

[18] D. T. B. Kelly, K. J. H. Law, And A. M. Stuart, Well-posedness and accuracy of the ensemble Kalman filter in discrete and continuous time, Nonlinearity, 27 (2014), pp. 2579-2603.

[19] Hui Hsiung Kuo, Gaussian measures in Banach spaces, Lecture Notes in Mathematics, Vol. 463, Springer-Verlag, Berlin, 1975.

[20] Peter D. Lax, Functional analysis, Pure and Applied Mathematics (New York), Wiley-Interscience [John Wiley \& Sons], New York, 2002.

[21] Alain Le Breton and Marie-Christine Roubaud, Asymptotic optimality of approximate filters in stochastic systems with colored noises, SIAM J. Control Optim., 39 (2000), pp. 917-927.

[22] F. Le Gland, V. Monbet, and V.-D. Tran, Large sample asymptotics for the ensemble Kalman filter, in The Oxford Handbook of Nonlinear Filtering, Dan Crisan and Boris Rozovskiǐ, eds., Oxford University Press, 2011, pp. 598-631.

[23] David M. Livings, Sarah L. Dance, and Nancy K. Nichols, Unbiased ensemble square root filters, Phys. D, 237 (2008), pp. 1021-1028.

[24] Jan Mandel, Loren Cobb, and Jonathan D. Beezley, On the convergence of the ensemble Kalman filter, Applications of Mathematics, 56 (2011), pp. 533-541.

[25] Józef Marcinkiewicz, Collected papers, Edited by Antoni Zygmund. With the collaboration of Stanislaw Lojasiewicz, Julian Musielak, Kazimierz Urbanik and Antoni Wiweger. Instytut Matematyczny Polskiej Akademii Nauk, Państwowe Wydawnictwo Naukowe, Warsaw, 1964.

[26] J. Marcinkiewicz And A. Zygmund, Sur les foncions indépendantes, Fund. Math., 28 (1937), pp. 60-90. Reprinted in 25], pp. 233-259.

[27] I. Mirouze And A. T. Weaver, Representation of correlation functions in variational assimilation using an implicit diffusion operator, Quarterly Journal of the Royal Meteorological Society, 136 (2010), pp. 1421-1443.

[28] Daniel Ocone and Etienne Pardoux, Asymptotic stability of the optimal filter with respect to its initial condition, SIAM J. Control Optim., 34 (1996), pp. 226-243.

[29] Olivier Pannekoucke, Lö̈k Berre, and Gerald Desroziers, Filtering properties of wavelets for local background-error correlations, Quarterly Journal of the Royal Meteorological Society, 133 (2007), pp. 363-379.

[30] Pavel Sakov and Peter R. Oke, Implications of the form of the ensemble transformation in the ensemble square root filters, Monthly Weather Review, 136 (2008), pp. 1042-1053.

[31] Dan Simon, Optimal State Estimation: Kalman, $\mathrm{H}_{\infty}$, and Nonlinear Approaches, John Wiley and Sons, 2006.

[32] Michael K. Tippett, Jeffrey L. Anderson, Craig H. Bishop, Thomas M. Hamill, and Jeffery S. Whitaker, Ensemble square root filters, Monthly Weather Review, 131 (2003), pp. 1485-1490.

[33] Xuguang Wang, Craig H. Bishop, and Simon J. Julier, Which is better, an ensemble of positive-negative pairs or a centered spherical simplex ensemble?, Monthly Weather Review, 132 (2004), pp. 1590-1605.

[34] J. S. Whitaker And T. M. Hamill, Ensemble data assimilation without perturbed observations, Monthly Weather Review, 130 (2002), pp. 1913-1924.

[35] Wojbor A. WoyczyŃski, On Marcinkiewicz-Zygmund laws of large numbers in Banach spaces and related rates of convergence, Probab. Math. Statist., 1 (1980), pp. 117-131 (1981).

[36] F. Zhang, Chris Snyder, And JuAnZhen Sun, Impacts of initial estimate and observation availability on convective-scale data assimilation with an ensemble Kalman filter, Monthly Weather Review, 132 (2004), pp. 1238-1253.

[37] J. F. Zhang, L. GuO, And H. F. Chen, L L-stability of estimation errors of Kalman filter for tracking time-varying parameters, International Journal of Adaptive Control and Signal Processing, 5 (1991), pp. 155-174. 